\title{
The effect of growth rate on formation of nitrogenous defects in diamond
}

Pal'yanov, Yu.N., Gusev, V.A., Kupriyanov, I.N., Borzdov Yu.M., Sokol, A.G., Khokhriakov, A.F., and Sobolev, N.V.

Institute of Mineralogy and Petrography, Siberian Branch of the Russian Academy of Sciences, Novosibirsk, 630090, Russia

\section{Introduction}

It is commonly accepted that nitrogen, the major impurity in diamond, incorporates into the diamond lattice as single substitutional atoms (C-centers), other forms of nitrogen (A-, B-centers) are formed as a result of high-temperature annealing during the diamond's mantle residence. Experimentally only the first stage of aggregation, namely conversion of the C-centers into the A ones has been intensively studied (Evans, 1992; Taylor et al., 1996). The results obtained are now widely used for evaluation natural diamond's mantle residence history. However, it was repeatedly pointed out that some peculiarities of the internal structure of diamond crystals can hardly be explained in terms of ordinary annealing (Sobolev, 1978; 1991).

It seems indubitable that primary factor for the formation of different intrinsic and impurity defects in diamond is the initial state of a crystal which is determined by the conditions of growth. As opposed to the high temperature annealing, the effect of the diamond's growth conditions (composition of crystallization medium, growth rate, initial concentration of nitrogen, etc.) on the formation of the nitrogenous defects is much less known.

The present work is devoted to the study the growth rate influence on the formation of $\mathrm{C}$ and $\mathrm{A}$ defects in diamond.

\section{Experimental}

Experimental study of diamond single crystals growth on seed by the temperature gradient method was carried out in the $\mathrm{Ni}-\mathrm{Fe}-\mathrm{C}$ and $\mathrm{Ni}-\mathrm{C}$ systems using high-pressure apparatus of split-sphere type BARS (Pal'yanov et al., 1990). The details of the growth techniques and methods of studying the crystals can be found in (Pal'yanov et al., 1997). Experiments in Ni-C system were conducted using crystallization scheme of "well" type. Diamond crystal growths on a seed of about $3 \mathrm{~mm}^{2}$ square in a well filled with the solvent catalyst. The cross-section of the well corresponds to the seed crystal shape. In this scheme, square of diamond growing on the seed is constant during all the growth period and only octahedral growth sector is present. All the experiments in Ni-C system were performed at temperature $1550^{\circ} \mathrm{C}$ with duration 20 hours.

\section{Results and Discussion}

Figure 1 shows the dependence of relative concentrations of the $\mathrm{A}$ and $\mathrm{C}$ centers on the growth rate, obtained for the crystals grown in $\mathrm{Ni}-\mathrm{Fe}-\mathrm{C}$ system. One can see that the lower growth rates the smaller fraction of $C$ centers and the large that one of A centers. The total nitrogen content $\left(\mathrm{N}_{\mathrm{A}}+\mathrm{N}_{\mathrm{C}}\right)$ in these crystals is on the average $200 \mathrm{ppm}$ and practically does not depend on the growth rate. The experiments on the crystal growth in the Ni-Fe-C system were performed at one temperature $\left(1500^{\circ} \mathrm{C}\right.$ ) but with different duration (at lower growth rates crystals were growing for a longer periods). So, the increase of the A centers concentration in the low growth rate samples could be due to the more prolonged annealing during the growth process. At present, the aggregation of single nitrogen atoms into the paired ones during high-temperature annealing is commonly described by the second order kinetics with rate equation

$$
K t=1 / C_{t}-1 / C_{0} \text {. }
$$

Using the rate constant values given in T. Evans's review (Evans, 1992) it is possible to estimate an annealing time required for achieving the observed levels of aggregation. For the crystal grown with the lowest over the series rate (mass rate $0.8 \mathrm{mg} / \mathrm{h}$ ) we obtain that the annealing time should be 
about 3700 hours whereas the growth time is 164 hours. So huge discrepancy clearly indicates that ordinary annealing could not be the major mechanism of the A centers formation during the diamonds growth.

It is known that the aggregation of nitrogen in diamond can be substantially enhanced by introduction of vacancies into a crystal (Collins, 1978). Beside this, recently it was shown (Fisher and Lawson, 1997) that incorporation of nickel and cobalt into synthetic diamonds also results in an increase in the aggregation rate, with the process of $\mathrm{C}$ to $\mathrm{A}$ transformation markedly deviating from simple second order kinetics. Since $\mathrm{Ni}$ and $\mathrm{Co}$ are the most common metals used in diamonds growth, the kinetics of nitrogen aggregation in synthetic diamonds and natural ones may be quite different.

To eliminate possible influence of annealing during the growth and clear up effect of the growth rate on the A centers formation a series of experiments with the same growth duration was carried out. It should be noted that in these experiments a "well" type growth scheme was employed which allowed diamonds with $\{111\}$ growth sectors only to be grown (to overcome the problem of growth sector dependence of nitrogen incorporation and aggregation). The results obtained are presented in Fig. 2. It is seen that the dependence of $N_{A} / N_{C}$ ration is qualitatively the same as shown in Fig. 1 , $i$. e. the lower growth rate the higher A centers content. Also of interest here is the increase of the total nitrogen content from $80 \mathrm{ppm}$ to $600 \mathrm{ppm}$ with decreasing the growth rate.

Now it is difficult to determine the exact mechanism of "grown in" A centers formation. At least three processes can be proposed:

a) pre-association of nitrogen atoms in crystallization medium;

b) diffusion of nitrogen along the surface of growing crystal;

c) nitrogen aggregation is impurity enhanced and incorporation of defects responsible for the enhancement depends strongly on growth rate

Whichever the process takes place, the results obtained in the present. work evidence that growth rate plays an essential role in the formation of nitrogenous defects in diamond. Beside this, taken in conjunction with results of Fisher and Lawson (Fisher and Lawson, 1997), they show that estimations of natural diamond's mantle residence time based on the nitrogen aggregation obeying simple second order kinetics may be incorrect.

This work was supported by the Russian Foundation for Basic Research, project no. 97-05-65195.

\section{References}

Collins, A. T., 1978, Migration of nitrogen in electron irradiated typed Ib diamond: J. Phys. C: Solid State Phys., 11, 10, p. L417-L422.

Evans, T., 1992, Aggregation of nitrogen in diamond. In The Properties of Natural and Synthetic Diamond (ed. J. Field), p. 295-290. Academic Press.

Fisher, D., and Lawson, S. C., 1997, The effect of nickel and cobalt on the aggregation of nitrogen in diamond. $8^{\text {th }}$ European Conf. on Diamond, Diamond-like and Related Materials - Heriot-Watt University, Edinburgh, Scotland. (to be published in Diamond and Related Materials)

Pal'yanov, Yu. N., et al., 1990, Growth of large diamond crystals using high pressure equipment of "BARS" type: Dokl. Acad. Nauk SSSR, 315, 5, 1221-1224. (in Russian)

Pal'yanov, Yu. N., et al., 1997, Growth conditions and real structure of synthetic diamond crystals: Russian Geology and Geophysics, 38, 5, p. 919-944

Sobolev, E. V., 1978, Nitrogen centers and growth of natural diamond crystals. In Problems of Petrology of the Earth's Crust and Upper Mantle, p. 245-255. Novosibirsk, Russia. (in Russian)

Sobolev, E. V., 1991, The impurity centers and some problems of diamond genesis. $5^{\text {th }}$ Intern. Kimberlite Conf. - Brasilia, Araxa. N2, p.388-390.

Taylor, W. R., Canil, D., and Milledge, H. J., 1996, Kinetics of Ib to IaA nitrogen aggregation in diamond: Geochimica et Cosmochimica Acta, 60, 23, p. 4725-4733. 
Fig. 1

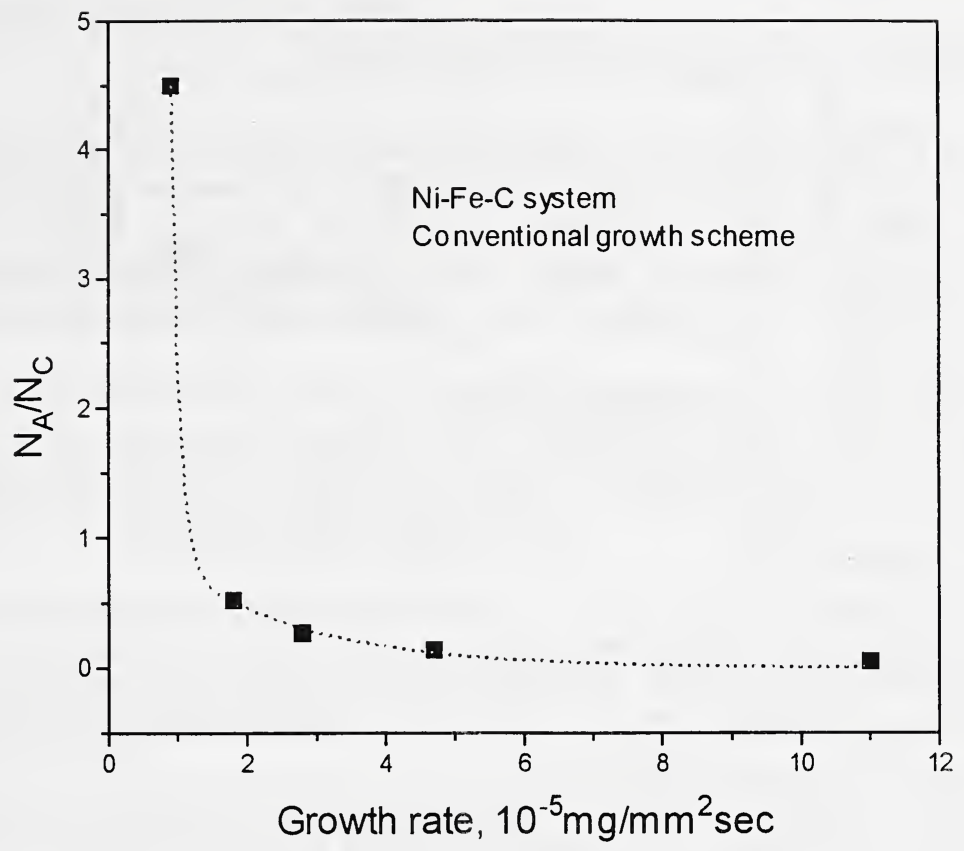

Fig. 2

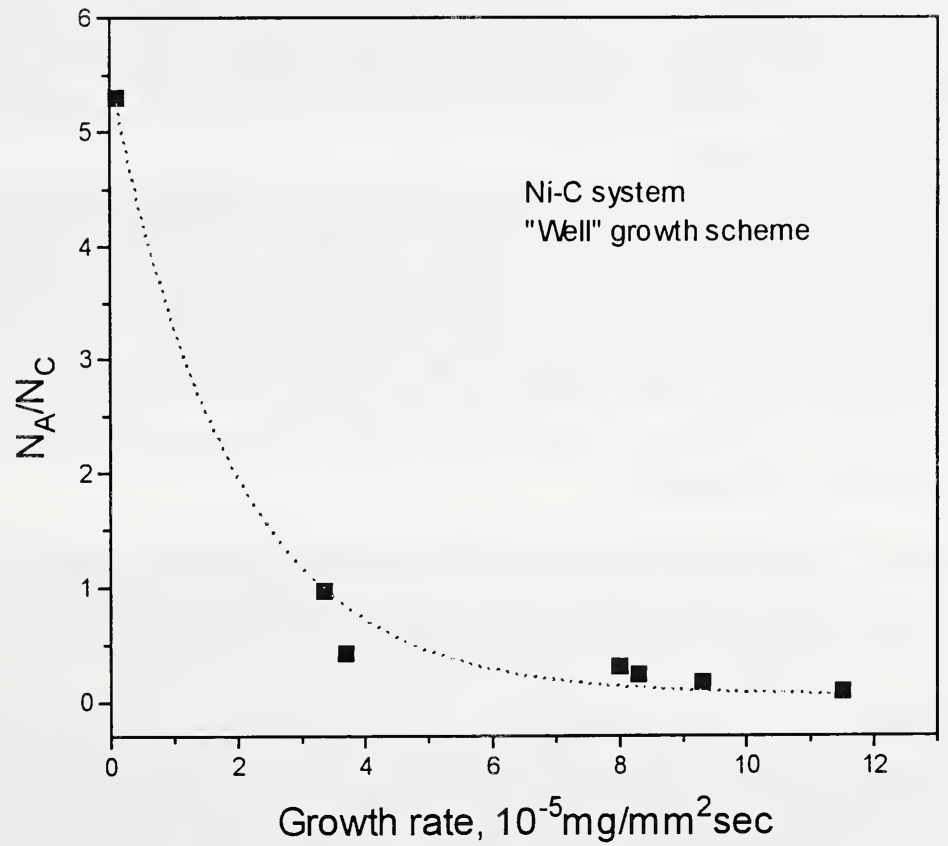

